\title{
Methicillin-Resistant Staphylococcus aureus Peritonitis due to Hematogenous Dissemination from Central Venous Catheter in a Maintenance Dialysis Patient
}

\author{
Gaetano Alfano a, b, c Monica Frisina ${ }^{a}$ Niccolò Morisi ${ }^{a}$ \\ Elisabetta Ascione $^{b}$ Francesco Fontana $^{b}$ Giacomo Morib \\ Caterina Cerami $^{d}$ Francesco Serra ${ }^{\text {Francesca Cabry }}{ }^{\mathrm{e}}$ \\ Decenzio Bonucchi $^{d}$ Roberta Gelmini ${ }^{e}$ Giovanni Guaraldi ${ }^{f}$ \\ Riccardo Magistroni ${ }^{a}$ b Gianni Cappelli ${ }^{a}$ b
}

aSection of Nephrology, Surgical, Medical and Dental Department of Morphological Sciences, University of Modena and Reggio Emilia, Modena, Italy; ${ }^{b}$ Nephrology Dialysis and Transplant Unit, University Hospital of Modena, Modena, Italy; ${ }^{\circ}$ Clinical and Experimental Medicine PhD Program, University of Modena and Reggio Emilia, Modena, Italy; ${ }^{d}$ Nephrology and Dialysis, Hospital Ramazzini-AUSL Modena, Carpi, Modena, Italy; eDepartment of General Surgery, University of Modena and Reggio Emilia, Modena, Italy; ${ }^{f}$ Clinic of Infectious Diseases, University Hospital of Modena, Modena, Italy

\section{Keywords}

Staphylococcus aureus · Peritonitis · Catheter · Dialysis · Infection · MRSA

\begin{abstract}
Staphylococcus aureus is a Gram-positive bacterium commonly associated with severe infections in hospitalized patients. S. aureus produces many virulence factors leading to local and distant pathological processes. Invasiveness of $S$. aureus generally induces metastatic infections such as bacteremia, infective endocarditis, osteomyelitis, arthritis, and endophthalmitis. Peritoneal localization from extra-abdominal infection can be a potential consequence of $S$. aureus infection. Two cases of metastatic peritonitis have been described in patients on peritoneal dialysis with concomitant peripheral vascular catheter-related bloodstream infection. We reported a case of peritoneal metastatic infection caused by methicillin-resistant Staphylococcus aureus (MRSA) in a patient on maintenance hemodialysis. A 37-year-old man was admitted with fever and chill due to jugular central vascular catheter (CVC)-related bloodstream infection caused by MRSA. CVC was placed after switching the patient from peritoneal dialysis to hemodialysis for scarce adherence to fluid restriction. Detection of MRSA on the peritoneal effluent combined with a total white blood cell count of 554 cells $/ \mathrm{mm}^{3} \mathrm{prompt}-$
\end{abstract}


ed the diagnosis of satellite MRSA peritonitis. Antibiotic treatment with daptomycin and simultaneous CVC and peritoneal catheter removal resolved the infectious process. No further metastatic localizations were detected elsewhere. In conclusion, S. aureus can induce metastatic infections far from the site of primary infection. As reported in this case, peritonitis can be secondary to the hematogenous dissemination of $S$. aureus especially in hospitalized patients having a central line.

(C) 2021 The Author(s).

Published by S. Karger AG, Basel

\section{Introduction}

Methicillin-resistant Staphylococcus aureus (MRSA) peritonitis is an uncommon and serious complication of peritoneal dialysis (PD). It is associated with substantial mortality and morbidity $[1,2]$. Generally, MRSA peritonitis is due to accidental contamination with skin bacteria during exchanges [3]. Secondary infection due to hematogenous dissemination is a rare cause of PD-related peritonitis [4]. We report a case of MRSA peritonitis secondary to a central venous catheter (CVC)-related bloodstream infection in a patient with end-stage renal disease on maintenance hemodialysis (HD).

\section{Case Report}

A 37-year-old man with end-stage renal disease of unknown origin started PD in June 2019 in a North African country. Scarce adherence to fluid restriction and frequent episodes of lung edema prompted a switch from PD to HD using a femoral venous catheter in August 2019. The peritoneal catheter was not timely removed and was left in place. After migration to Southern Italy, the patient was hospitalized in September 2019. Here, the femoral catheter was replaced by a tunneled jugular CVC, and an arteriovenous fistula was created as a future vascular access for HD. The peritoneal catheter was left in place because of the patient's frequent movements in Italy.

In September 2020, the patient was admitted to our hospital for hyperpyrexia (temperature of $38^{\circ} \mathrm{C}$ ) with chills. At presentation, the patient was alert and normally oriented (Glasgow Coma Scale 15/15); he had a peripheral arterial blood pressure of 147/83 $\mathrm{mmHg}$ and normal oxygen saturation. Chest X-ray did not reveal any lung abnormalities and nasopharyngeal swab for SARS-CoV-2 resulted negative. Laboratory evaluation showed elevated levels of procalcitonin $(283 \mathrm{ng} / \mathrm{mL})$ and C-reactive protein $(8 \mathrm{mg} / \mathrm{dL})$. The abdomen was soft without tenderness or organomegaly at physical examination. No signs of peritoneal catheter exit site or tunnel infection were detected. Blood culture (from central line and peripheral sites) and peritoneal effluent culture were performed before starting of empirical IV antibiotic with piperacillin/tazobactam for the suspicion of bacterial infection. The patient tested negative for MRSA nasal-swab screening.

Blood cultures collected from CVC became positive after $5 \mathrm{~h}$ and $4 \mathrm{~min}$, whereas blood cultures collected from two peripheral sites became positive for the same bacterium after 1 day, $9 \mathrm{~h}$ and $41 \mathrm{~min}$ in one site and after $15 \mathrm{~h}$ and $48 \mathrm{~min}$ in another site. The time to positivity of blood culture, defined as the time between the start of incubation and the time of growth in the culture bottle, suggested a catheter-related infection. The same antibiotic-spectrum MRSA was also detected on the peritoneal effluent. The diagnosis of peritonitis was supported by the detection of elevated leukocyte count ( 544 cells $/ \mathrm{mm}^{3}$ with $76 \%$ of neutrophils) on the peritoneal effluent.

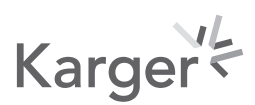


Case Reports

in Nephrology and Dialysis

Table 1. Antibiotic-susceptibility testing of $S$. aureus

\begin{tabular}{l|l}
\hline Case Rep Nephrol Dial 2021;11:281-285 \\
\hline DOI: 10.1159/000517143 & $\begin{array}{l}\text { ○ 2021 The Author(s). Published by S. Karger AG, Basel } \\
\text { www.karger.com/cnd }\end{array}$ \\
\hline
\end{tabular}

Alfano et al.: Metastatic MRSA Peritonitis in a Dialysis Patient

\begin{tabular}{lll}
\hline Antibiotic & Interpretation & $\mathrm{MIC}, \mu \mathrm{g} / \mathrm{mL}$ \\
\hline Penicillin G & $\mathbf{R}$ & $\geq 0.5$ \\
Oxacillin & $\mathbf{R}$ & 2 \\
Erythromycin & $\mathrm{S}$ & 1 \\
Clindamycin & $\mathrm{S}$ & 0.5 \\
Rifampicin & $\mathrm{S}$ & Lowest \\
Levofloxacin & $\mathrm{S}$ & 0.25 \\
Trimethoprim/sulfamethoxazole & $\mathrm{S}$ & Lowest \\
Tetracycline & $\mathbf{R}$ & $\geq 16$ \\
Tigecycline & $\mathrm{S}$ & Lowest \\
Gentamicin & $\mathbf{R}$ & $\geq 16$ \\
Teicoplanin & $\mathrm{S}$ & Lowest \\
Vancomycin & $\mathrm{S}$ & 1 \\
Linezolid & $\mathrm{S}$ & 2 \\
Daptomycin & $\mathrm{S}$ & 0.25 \\
Mupirocin & $\mathrm{S}$ & Lowest \\
\hline
\end{tabular}

$\mathrm{R}$, resistant; S, susceptible.

The not recent use of the peritoneal catheter and lack of clear symptoms of peritonitis was consistent with metastatic dissemination of MRSA to the peritoneum. Based on these findings, the patient underwent replacement of colonized jugular CVC and peritoneal catheter removal. Specific antimicrobial therapy with daptomycin (500 mg every 2 days) was administered on the basis of antibiotic-susceptibility testing (Table 1). After 15 days of antibiotic therapy from the last negative blood cultures, the patient was discharged asymptomatic and without echocardiographic findings of endocarditis. He continued thrice-weekly HD treatments with arteriovenous fistula after jugular CVC removal.

\section{Discussion}

S. aureus is a major bacterial human pathogen able to cause a wide spectrum of potentially severe manifestations, especially in patients with a history of recent hospitalization and temporary catheter as a dialysis access [5]. Emergence of multidrug resistant strains such as MRSA is a challenge in treating invasive infections including bacteremia, infective endocarditis, osteomyelitis, arthritis, and infection of prosthetic devices [6]. S. aureus pathogenicity is due to multiple virulence factors that drive local damage and metastatic spread of bacteria from a primary site. Secondary endophthalmitis [7, 8], vertebral osteomyelitis [9], and endocarditis [10] are classical metastatic infections of $S$. aureus bacteremia. Hematogenous dissemination to the peritoneum from a peripheral or central intravenous catheter is an uncommon but potential source of infection for patients on PD. Ma et al. [4] reported S. aureus peritonitis secondary to bacteremia originating from peripheral catheters placed for intravenous infusion in hospitalized patients. Based on these findings, patients on PD might be at high risk of metastatic $S$. aureus peritonitis from distant foci (i.e., peripheral or central intravenous devices). It is worth noting that $S$. aureus peritonitis is a severe form of Gram-positive peritonitis [11] because it is often associated with hospitalization, peritonitis relapse, catheter removal, PD failure, and mortality [12,13].

In conclusion, interventions that reduce the risk of MRSA bloodstream infections, such as care for skin lesions, avoidance of long-term CVC, fast removal of the peritoneal catheter, and 
eradication of nasal carrier of MRSA $[14,15]$ may reduce the risk of bacteremia with metastatic infections.This case report shows that the peritoneum may be a potential site of $S$. aureus metastatic infection. The consequences of peritoneum infection could be even more severe for the patients on PD maintenance because glucose-based dialysate solution promotes microbial growth and can facilitate biofilm formation on the peritoneal catheter. Hence, detection of $S$. aureus bacteremia should prompt adequate antibiotic therapy in order to limit the severe consequences of this antibiotic-resistant pathogenic bacterium.

\section{Acknowledgments}

The authors thank all health workers involved in the care of this patient from his arrival in Italy.

\section{Statement of Ethics}

The Ethics Committee of the University Hospital of Modena does not require ethical approval for reporting individual cases. Written informed consent was obtained from the patient for publication of this case report.

\section{Conflict of Interest Statement}

The authors declare no potential conflicts of interest with respect to the research, authorship, and/or publication of this article.

\section{Author Contributions}

G.A., M.F., and N.M. contributed equally to this work. G.A. and E.A. designed the manuscript; M.F. and N.M. wrote the first draft of the manuscript; E.A., F.F., G.M., C.T., F.R., and F.C. furnished data of the patient; D.B., R.G., G.G., R.M., and G.C. revised the manuscript critically. All authors reviewed and edited the manuscript and approved the final version of the manuscript.

\section{References}

1 Szeto CC, Chow KM, Kwan BC, Law MC, Chung KY, Yu S, et al. Staphylococcus aureus peritonitis complicates peritoneal dialysis: review of 245 consecutive cases. Clin J Am Soc Nephrol. 2007 Mar;2(2):245-51.

2 Govindarajulu S, Hawley CM, McDonald SP, Brown FG, Rosman JB, Wiggins KJ, et al. Staphylococcus aureus peritonitis in australian peritoneal dialysis patients: predictors, treatment, and outcomes in 503 cases. Perit Dial Int. 2010 Jan;30(3):311-9.

3 Miller TE, Findon G. Touch contamination of connection devices in peritoneal dialysis: a quantitative microbiologic analysis. Perit Dial Int. 1997 Nov;17(6):560-7.

4 Ma MKM, Yap DYH, Yip TPS, Lui SL, Lo WK. Staphylococcus aureus peritonitis in two peritoneal dialysis patients: an uncommon complication of peripheral intravenous catheter infection. Perit Dial Int. 2012;32(5):573-4.

5 Jensen AG, Wachmann CH, Poulsen KB, Espersen F, Scheibel J, Skinhøj P, et al. Risk factors for hospital-acquired Staphylococcus aureus bacteremia. Arch Intern Med. 1999 Jul;159(13):1437.

6 Turner NA, Sharma-Kuinkel BK, Maskarinec SA, Eichenberger EM, Shah PP, Carugati M, et al. Methicillin-resistant Staphylococcus aureus : an overview of basic and clinical research. Nat Rev Microbiol. 2019 Apr;17(4):203-18.

7 Bhavsar MM, Devarajan TV, Nembi PS, Mani AK, Mani AK. Metastatic endogenous endophthalmitis: a rare presentation with methicillin-resistant Staphylococcus aureus prostatic abscess. Indian J Crit Care Med. 2017 Mar;21(3):172-5. 
8 Steeples LR, Jones NP. Staphylococcal endogenous endophthalmitis in association with pyogenic vertebral osteomyelitis. Eye. 2016 Jan;30(1):152-5.

9 Jensen AG, Espersen F, Skinhøj P, Frimodt-Møller N. Bacteremic Staphylococcus aureus spondylitis. Arch Intern Med. 1998 Mar;158(5):509.

10 Røder BL, Wandall DA, Frimodt-Møller N, Espersen F, Skinhøj P, Rosdahl VT. Clinical features of Staphylococcus aureus endocarditis: a 10-year experience in Denmark. Arch Intern Med. 1999 Mar;159(5):462.

11 Szeto CC, Li PK, Johnson DW, Bernardini J, Dong J, Figueiredo AE, et al. ISPD catheter-related infection recommendations: 2017 update. Perit Dial Int. 2017 Mar;37(2):141-54.

12 Zimmerman SW, O'Brien M, Wiedenhoeft FA, Johnson CA. Staphylococcus aureus peritoneal catheter-related infections: a cause of catheter loss and peritonitis. Perit Dial Int. 1988 Jul;8(3):191-4.

13 Govindarajulu S, Hawley CM, McDonald SP, Brown FG, Rosman JB, Wiggins KJ, et al. Staphylococcus aureus peritonitis in Australian peritoneal dialysis patients: predictors, treatment, and outcomes in 503 cases. Perit Dial Int. 2010 May;30(3):311-9.

14 Lye WC, Leong SO, Lee EJ. Methicillin-resistant Staphylococcus aureus nasal carriage and infections in CAPD. Kidney Int. 1993 Jun;43(6):1357-62.

15 Lai CF, Liao CH, Pai MF, Chu FY, Hsu SP, Chen HY, et al. Nasal carriage of methicillin-resistant Staphylococcus aureus is associated with higher all-cause mortality in hemodialysis patients. Clin J Am Soc Nephrol. 2011 Jan; 6(1):167-74. 